\title{
Criminologie
}

\section{Cent ans de prison : les conditions et les "privilèges " des détenus hommes, femmes et enfants}

\section{Jacques Laplante}

Volume 24, numéro 1, 1991

Les droits des détenu-e-s

URI : https://id.erudit.org/iderudit/017300ar

DOI : https://doi.org/10.7202/017300ar

Aller au sommaire du numéro

Éditeur(s)

Les Presses de l'Université de Montréal

ISSN

0316-0041 (imprimé)

1492-1367 (numérique)

Découvrir la revue

Citer cet article

Laplante, J. (1991). Cent ans de prison : les conditions et les « privilèges " des détenus hommes, femmes et enfants. Criminologie, 24(1), 11-32.

https://doi.org/10.7202/017300ar
Résumé de l'article

The creation of the prison in the xixth century cannot be dissociated from the misery and distress it inflicts on a group of men, women and children who are already (in various ways) in a minority position in society. Reform after reform of Quebec prisons over more than a hundred years have not created a milieu without this misery and distress as though these were an inherent part of prison life itself. How, then, explain the persistance in wanting to keep such an institution whose objective was the moral rectification of the individual and insisted on rehabilitating him ? 


\section{CENT ANS DE PRISON: \\ LES CONDITIONS ET LES «PRIVILĖGES» DES DÉTENUS HOMMES, FEMMES ET ENFANTS \\ Jacques Laplante*}

The creation of the prison in the XIXth century cannot be dissociated from the misery and distress it inflicts on a group of men, women and children who are already (in various ways) in a minority position in society. Reform after reform of Quebec prisons over more than a hundred years have not created a milieu without this misery and distress as though these were an inherent part of prison life itself. How, then, explain the persistance in wanting to keep such an institution whose objective was the moral rectification of the individual and insisted on rehabilitating him?

Au moment où le pénal était en pleine construction, la prison apparaissait comme un des plus grands espoirs de réformer les hommes, femmes et enfants qui ont pour principale caractéristique de n'avoir ni lieu ni feu. La prison aura vite fait de montrer ses vices et son incapacité à corriger des individus le plus souvent victimes, par ailleurs, de conditions sociales difficiles. Pourtant, elle est très vite donnée comme remède à elle-même. Le pénitencier, l'asile, l'école de réforme et d'industrie ne sont pas étrangers à cette première prison pénale. Dans ce travail, toutefois, seuls la prison et le pénitencier au Québec et au

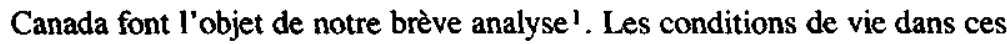
lieux de sûreté et les quelques privilèges que les individus y ont gagnés en cent ans guident notre réflexion sur cet instrument de réforme.

\section{LA PRISON À SES DÉBUTS}

Dans plusieurs pays européens mais aussi en Amérique du Nord, des bouleversements importants surviennent dans la vie sociale et économique, au début du XIX ${ }^{e}$ siècle. Au Canada, les récriminations des marchands, la poussée des immigrants ne sont pas sans influencer la mutation assez soudaine que connaît l'usage des prisons. Les cris à l'oisiveté, au vice constituent un autre

* Professeur, Département de criminologie, Université d'Ottawa, Pavillon Tabaret, Ottawa (Ontario) K1N 6N5.

1. Pour une analyse plus approfondie de la genèse de la prison et du pénitencier au Québec et au Canada, de leur mise en place et leur maintien en égard aux autres institutions sociales, voir Jacques Laplante, Prison et ordre social au Québec (1989), aux Presses de l'Université d'Ottawa. 
facteur favorisant l'importation de l'idée d'emprisonnement comme peine.

Cette prison semble être le fruit d'une nouvelle civilisation, en même temps qu'elle aide à la former. Comme elle ne laisse que rarement des marques apparentes sur sa «clientèle», elle est vite «acceptée comme une innovation à perfectionner plutôt qu'à contester» (Foucault, 1980, p. 30). Cette idée de substitution de la prison aux anciennes tortures apparaît comme une amélioration. De fait, la prison vient plutôt compléter la nouvelle fonction sociale d'un État qui est désormais moins considéré comme un ensemble disparate d'institutions de soutien à la domination seigneuriale et marchande, que comme un appareil en voie d'intégration, de centralisation et de spécialisation. Cet appareil cherchera à prendre sous sa charge l'ensemble des «crimes", sans tenir compte des moyens locaux de médiation et de solution des conflits.

À Québec, par exemple, les crimes contre la propriété inquiètent, d'autant plus qu' ils augmentent de façon constante, de 1814 à 1834, alors que les délits contre la personne et contre la justice restent d'une relative stabilité. Mais de 1825 à 1832, le nombre de crimes faisant l'objet d'un internement grimpe brusquement. C'est dans la catégorie des crimes contre l'ordre public qu'il faut chercher la cause de la montée des détentions, de dire Fecteau (1980, p. 19). En 1823-1825, l'émeute et l'exploitation d'une «maison de désordre» sont en tête des chefs d'accusation. En 1832-1834, ces délits cèdent la place à l'oisiveté, comme cause première d'emprisonnement. En effet, le délit de vagabondage compte pour 36 pour cent de tous les chefs d'emprisonnement, en 1832-1834, alors que ce rapport était de 6 pour cent, en 1814-1817, et de 3 pour cent, en 1823-1825.

Qui tente ainsi de contrer le vagabondage? Pour un temps, seuls un ou deux juges s'en chargent pour tout le district, mais déjà, de 1824 à 1833 , le nombre de juges de paix actifs double (passant de 21 à 41).

La prison devient un instrument de peine. Les sentences rendues par les juges sont uniformisées: la condamnation à la maison de correction (qui forme un seul et même édifice avec la prison, à cette époque) devient systématique (en 1832-1834, 81 pour cent des condamnés sont envoyés à la maison de correction).

Mais comment corrige-t-on ces vagabonds dans ces lieux de la prison qu'on appelle maisons de correction? En réalité, la prison ne remplit aucunement son rôle de correction. De fait, elle constitue, dès ses débuts, un lieu de perdition. Tentons de constater, quoique bien partiellement, ce qui s'y passe.

En 1837, François-Réal Angers, rapporteur des débats à la Chambre, visite la prison de Québec et en fait une description: 
Chaque étage est divisé en deux par un passage ou corridor, aux deux côtés duquel sont les chambres des prisonniers. Chaque chambre peut avoir environ 15 pieds carrés, et contenir 12 ou 15 personnes. Il y a autour de cet appartement commun de petites cellules, qui servent de cabinet de nuit pour deux ou trois prisonniers.

La première chambre que nous visitâmes renfermait les criminels condarnnés récemment à la déportation: ils étaient au nombre de 13, tous dans la fleur de l'âge et condamnés pour récidive, le plus jeune n'avait que 12 ans, le plus âgé n'en avait pas 25 (...)

(...) de cette chambre nous passâmes dans celle des malheureux insensés, qui parcourent nos rues l'été, et que la police empêche ainsi de périr pendant l'hiver, en les enfermant dans une prison à défaut d'un asile que nous n'avons pas (1837). C'était la réunion qui offrait le tableau le plus affligeant et le plus désagréable qu'il y eût dans ce bâtiment et qui portait surtout l'empreinte de la misère et de la dégradation.

(...) Nous visitâmes enfin le cachot des condamnés. En entrant dans cet asile, nous aperçûmes quatre hommes, étendus sur un méchant grabat, et éclairés par une seule lampe, qui ne jetait dans cette étroite demeure qu'une faible clarté (...) Il n'était guère possible d'entrer dans ce cachot étroit, bas et obscur, et d'aborder ces quatre personnes, destinées à une mort honteuse, et dont la pâleur était augmentée encore par la teinte jaunâtre des murailles, sans éprouver une émotion vive, un serrement de cœur. (Angers, cité dans Boyer, 1966, p. 447.)

Le révérend Sewell décrit sous un autre angle cette prison de Québec (Sewell, in Durham, 1838, Appendix to Report on the Affairs of British North America, pp. 14-15). D'abord, elle est en pleine ville, compliquant ainsi la vigilance et la discipline que devraient y apporter ses officiers. Y sont destinées toute une variété de personnes : les condamnés, ceux qui y attendent leur procès, les débiteurs infortunés, les maniaques, les vilains endurcis, les jeunes. Pendant l'année 1830, 743 personnes occupèrent la prison, dont 170 femmes, 60 jeunes (moins de 20 ans), 70 récidivistes et 235 vagabonds. Parmi ceux-ci, au moins la moitié cherchaient refuge dans la prison pour éviter de mourir de faim ou de froid. Les dépenses de la prison pour cette même année étaient d'environ 1770 livres.

Le révérend signale, à titre de secrétaire honorifique de l'Association de la prison, que la création d'un pénitencier avait été suggérée à l'Assemblée, en 1829-1830, afin de faciliter la séparation des prisonniers. De même, il indique qu'une maison d'industrie pourrait être utile pour les prisonniers qui, à la fin de leur sentence, désirent se réadapter au travail. Cette maison pourrait encore desservir les vagabonds et les enfants des prisonniers. 
Compte tenu de la nature physique des lieux décrits par Angers, il est clair que tous les prisonniers se retrouvent ensemble, aussi bien le jour que la nuit. Et il y a, de plus, les femmes qui se trouvent dans la dite maison de correction, à l'intérieur de la même prison.

Des récidivistes, des femmes, des insensés, des vagabonds, des condamnés, des jeunes; voilà ce dont se compose surtout la population de la prison de Québec.

À la même époque, ce qui se passe à la prison de Montréal est bien à l'image du désceuvrement connu à la prison de Québec. On y trouve aussi un ramassis de toutes sortes d'infortunés (Grand Jury, Journaux de la Chambre d'Assemblée de la province du Bas-Canada, vol. XIV, et Comité pour enquêter sur l'institution du système pénitencier au Canada, 1836).

À la prison de Montréal, l'incarcération mixte des hommes et des femmes attire, en 1835-1836, l'attention des grands jurés. C'est d'ailleurs le seul sujet dont ils veulent entretenir la Cour, car, faut-il le dire, les qualités morales des femmes concernant tout ce qui a rapport aux questions sexuelles precoccupent sans cesse les autorités:

Elles sont abandonnées à elles-mêmes, vivant dans l'oisiveté et s'encourageant mutuellement au vice. Elles ne sont surveillées que par des hommes. (Bas-Canada, Journaux de la Chambre d'Assemblée de la province du Bas-Canada, vol. 45, 1836.)

Les grands jurés demandent à la Cour d'adopter pour la prison de Montréal le modèle suivi à Québec, où les femmes sont sous la surveillance d'une femme «respectable» qui les fait travailler et qui leur donne leçons et exemples de vertu.

Car il en faut de la vertu pour demeurer dans une cellule où l'on a peine à se retourner et où il n'y a ni paillasses, ni couvertures. Il n'en faut pas moins pour se contenter, pour toute nourriture, d'une livre et demie de pain et d'un gallon d'eau par jour et encore, seulement si on le gagne en travaillant et en étant docile (Borthwick, cité par Lefebvre, 1954, p. 527).

De fait, c'est la mort, de faim et de froid, d'un vagabond, en 1835 , qui permet d'entrevoir les conditions impossibles de la prison dans l' 'enquête générale» ordonnée à ce moment. Le geôlier et ses fils (ivrognes, débauchés, vagabonds reconnus) seraient les principaux responsables de la corruption, du traitement abusif des prisonniers et de leur mort (principalement chez les vagabonds) (Bas-Canada, Journaux de la Chambre d'Assemblée, 1836). 
En effet, les enquêteurs en arrivent à la conclusion que, malgré le mauvais état de la prison, ceux qui en ont la garde peuvent chauffer et nourrir correctement les prisonniers et prisonnières.

Pourtant en 1857, cette prison n'a guère changé:

On pourrait (la prison) presque l'appeler une maternité, tant sont nombreuses les femmes enceintes qui y viennent, qui y font leurs couches (...) On pourrait la nommer un hospice pour les enfants qui y sont reçus en nombre très considérable, et à un âge très tendre (...) Un hospice pour les individus âgés des deux sexes, et pour les infirmes de tous les genres (...) un asile d'aliénés (...) (Lettre du médecin Beaubien à l'inspecteur Nelson, cité dans Boyer, 1966, p. 482.)

Même si, selon les inspecteurs en 1859 , les 30 pour cent de femmes qui constituent la population des prisons communes sont quasi toutes des prostituées, il y a plusieurs centaines ( 600 environ) de jeunes en bas de 16 ans (garçons et filles), des récidivistes de toutes sortes, des ivrognes, etc. La prison ramasse, de plus, tous ceux qui traînent dans les rues et qui gênent. Elle continue d'ailleurs à le faire bien au-delà de ses premières expériences.

\section{LA VOCATION DES PRISONS ET LEUR RÔLE EFFECTIF}

Les inspecteurs, qui reçoivent leur mandat en 1868 , font dorénavant des visites dans les prisons et soumettent un rapport annuel. Ils nous instruisent non seulement sur la vocation avouée de la prison, mais encore sur son rôle effectif.

Selon les inspecteurs, qui remplit ces prisons? Pour la grande partie, des infortunés sans éducation, perdus par des parents «sans entrailles» ou des amis corrompus, abandonnés. Les prisons deviendraient pour eux d'agréables refuges.

Les inspecteurs au Québec, dans leur premier rapport, racontent ce qu'étaient les prisons, en $\mathbf{1 8 5 8}$. Ils le font en rapportant le bilan des inspecteurs de prisons du Haut et du Bas-Canada. Rien, absolument rien ne va:

Les défauts de nos Prisons sont de tous les genres, et bien qu'il y ait divers degrés, il n'en reste pas moins vrai qu'aucun de ces établissements ne répond au triple but qu'on doit se proposer en les formant, savoir : punir, contenir et amender. (Document de la Session, $\mathrm{n}^{\circ} 23,1869$, pp. 3-4.)

Les prisons ont été négligées à ce point que dans presque toutes, il n'existe aucune espèce de règlements; les choses vont comme elles peuvent, et le malheureux geôlier est forcé d'en prendre son parti et de se tirer 
d'affaire sans l'autorité du Shérif qui n'est guère mieux situé que lui sous ce rapport. (Document de la Session, $\mathrm{n}^{\circ} 23,1869$, pp. 3-4.)

Les défauts se situent au niveau de la surveillance, de la discipline, des moyens de moralisation, du personnel, des constructions et de l'hygiène. Six prisons n'auraient que le geôlier pour tout faire, alors que les règlements sont inexistants, que la classification des prisonniers est impossible.

Les prisonniers demeurent ensemble pendant le jour, et couchent ensemble (à peu d'exceptions près) par deux, par trois et par six pendant la nuit. En dehors de la séparation plus ou moins parfaite des jeunes, on peut dire que les âges, les conditions, les moralités forment dans ces établissements, un pêle-mêle déplorable au milieu duquel nous apparaissent de pauvres aliénés, de malheureux indigents sans feu ni lieu, et de plus malheureuses créatures encore, qu'une première faute, souvent comparativement légère, condamne presque infailliblement de cette sorte à une perte complète. (Document de la Session, $\mathrm{n}^{\mathbf{0}} 23,1869$, pp. 3-4.)

En plus d'être défectueuses, les prisons varient dans leur degré de médiocrité : d'une discipline à peine apparente à une discipline nulle, d'un logement passable à un logement affreux, d'un travail sporadique à l'oisiveté complète (même là où le tribunal a imposé les travaux forcés au condamné), d'infractions à la discipline punies à aucune infraction notée parce qu'aucune discipline et de ce fait aucune punition. De plus, rien n'est fait pour pourvoir aux «besoins religieux ", de sorte qu'il n'y a pas de moralisation possible.

Bref, «il faut se hâter de le dire à la suite de beaucoup d'officiers, d'aumôniers et de médecins de nos Institutions pénales, nos Prisons communes sont des écoles de vice où dans le sein de l'indolence et des voluptés de la crapule, les jeunes délinquants vont apprendre les roueries du mal, de ceux qui, plus vieux dans le crime, s'en constituent les précepteurs et les modèles" (Document de la Session, $\mathrm{n}^{\circ} 23,1869$, pp. 3-4).

Les objectifs de la prison ne sont pas atteints faute de personnel, d'hygiène, de surveillance, de règlements, de religion et de la moralisation; c'est là le message de ces inspecteurs. Même la clientèle n'est pas bien choisie, car c'est aussi elle qui doit assurer le succès de l'institution.

Le choix de la clientèle semble très important et cet élément vient compléter la kyrielle des fameuses maximes des prisons (Foucault, 1975, pp. 274-275). Changer le comportement, classer, individualiser la peine, faire travailler, éduquer, surveiller (techniquement) prennent leur importance alors que la relance se fait déjà souvent sous le signe de la charité. 
Que sont devenues ces prisons dix ans plus tard? Selon les inspecteurs, on y trouve des gardiens un peu plus zélés, à cause des règlements mis en vigueur, un classement des prisonniers un peu plus soigné, des livres de dépenses mieux tenus. Mais ce sont surtout les défauts qu'ont retenus les prisons, que ce soit dans les «moyens de moralisation», dans l'hygiène ou dans la construction elle-même.

Il faudrait séparer les femmes des hommes, organiser le travail, établir des prisons centrales, faire l'instruction religieuse, aménager les «lieux d'aisances». Enfin, pour mettre un peu d'ordre, il faudrait établir un plan d'ensemble des maisons pénales et de bienfaisance qui soit capable de distinguer les différences dans le mal, le contrer ou le prévenir: séparer les hommes des femmes, oui, mais aussi les jeunes des vieux et des vieilles et les diviser aussi entre eux, isoler les fous, distinguer protégés et délinquants, éloigner les alcooliques, ranger les récidivistes, s'occuper des pauvres honnêtes, etc. Le vice dans la grande majorité des cas et les vertus morales (associées au sexe) des femmes à chaque occasion reviennent diriger les contours du réseau.

L'espoir des inspecteurs qui proposent ce réseau est, ni plus ni moins, de réserver la prison aux cas spécifiques. Les cinq mille prisonniers détenus en moyenne annuellement pourraient être mieux contenus pour arrêter la criminalité, le rêve d'une bonne classification se réaliserait enfin, la récidive serait éliminée et le travail des prisonniers amélioré. Cela viendrait changer l'image que leur laisse la population carcérale des 12 dernières années alors que la récidive est fréquente, le nombre de fous emprisonnés inadmissible, le travail, en plus de ne rapporter qu'une fraction du coût de la prison (65 cents par prisonnier, en moyenne, non en une année mais bien en 10 ans), insignifiant en soi. Pourtant, la prison apparaît nécessaire à une bonne administration de la justice, au moins autant que le tribunal.

Ce n'est qu'en 1903 que les inspecteurs s'avisent que «les récidivistes ne sont pas toujours des criminels endurcis», mais souvent des gens sans travail qui cherchent à passer l'hiver sous un abri quelconque. Ce changement notable de point de vue en rapport avec les récidivistes s'ajoute à un autre constat des inspecteurs: la montée de la population en prison peut être due à des «institutions respectables» dont ils ne peuvent critiquer le rôle. Ils ne révèlent pas quelles sont ces institutions.

Le parcours suivi par les inspecteurs, pendant un demi-siècle, a abouti à un cul-de-sac, aussi bien pour eux que pour les quelques idées qu'ils ont défendues. En effet, leur démission semble imminente vers la fin du siècle dernier et leur rôle s'efface lentement. Ils ne seront là que pour rapporter des incidents et serviront moins de caution aux devoirs que l'État disait devoir remplir. 
En réalité, on peut affirmer que les inspecteurs n'ont guère vu la prison s'améliorer dans leurs nombreuses visites, si ce n'est au niveau de certaines conditions de vie. Leurs visites étaient par trop rapides, et pour cause. Pensons simplement au nombre d'institutions à inspecter par un si petit nombre d'inspecteurs.

C'est sur les récidivistes que s'acharnèrent principalement les inspecteurs. Ceux-ci s'aperçurent, après quelque trente ans, que bon nombre de ces récidivistes pouvaient bien être sans malice et ne représenter aucun danger. N'empêche que c'est à partir d'eux qu'ils ont aidé l'État à construire la prison. Au second rang viennent les femmes. Jamais ils ne se sont lassés de les décrier, sur la base de leur habitude de la prostitution et de la dégradation morale où les plonge ce métier. Le récidiviste aussi bien que la femme déchue ont ainsi permis de réaffirmer les valeurs sociales. Les inspecteurs se devaient de faire appel à ces valeurs. C'est par elles que l'on passe pour construire la prison et pour en faire valoir les maximes. Celles-ci, poursuivies sans relâche par les inspecteurs, montrent bien le peu de distance qu'ils ont prise par rapport à cette prison, celle-ci échouant sans cesse à atteindre ses objectifs déclarés.

Tout compte fait, le discours des inspecteurs, fait d'espoirs, de peurs, de dénonciations, d'encouragements, de dépits, de mythes, s'est inspiré de leurs observations d'un groupe de vagabonds et d'ivrognes pauvres (hommes, femmes ou enfants).

En 1871, des 3640 offenses commises par les détenus, le vagabondage et l'ivrognerie comptent pour 1605 . Les offenses graves ne totalisent pas plus d'une trentaine. Vers la fin du siècle, en 1898, l'on trouve encore l'ivrognerie et le vagabondage, cette fois pour les trois quarts des 4370 offenses, alors que les offenses graves ne comptent encore que pour une trentaine.

Ce même discours, et ce que l'on a bien voulu en croire (car des vagabonds et des ivrognes peuvent déranger, mais ne présentent habituellement aucun danger), a servi à monter un appareil fabuleux sans aucune proportion avec les problèmes réels.

Malgré cette immense misère qui se rencontre dans la prison et malgré aussi cette énorme cruauté que constitue la prison en elle-même, on le sait, une autre est proposée: le pénitencier. On se persuade alors qu'elle peut être un remède à elle-même et qu'elle est nécessaire pour contrer le «crime grandissant». Elle est d'abord expérimentée chez nos voisins du sud, en tant que système bien entendu, avant d'apparaître chez nous. 


\section{LE PREMIER PÉNITENCIER}

Aux États-Unis d'Amérique, deux grands systèmes apparaissent. Cherry Hill impose sa discipline par les limites de la cellule, qui telle une cage s'appesantit sur l'individu, le disciplinant avant même qu'il ne commette une infraction aux règles du pénitencier. Dans le système auburnien, c'est le fouet (instrument privilégié), la solitude absolue, la privation de nourriture, de sommeil, etc., qui font respecter les exigences de la vie pénitentiaire, soit le travail et le silence.

Ces deux systèmes pénitentiaires fort similaires animent les débats politiques pendant plus de cinquante ans aux États-Unis (et ailleurs). C'est dire l'importance du «criminel», c'est confirmer la valeur de l'ordre que l'on veut instaurer. Le «criminel», c'est un ennemi du genre humain et il a contre lui l'humanité tout entière. Dans la pensée de la démocratie américaine, «il est une déviance destructrice de la norme, de ce consensus moteur du fonctionnement global» (Introduction aux auvres de Tocqueville, par M. Perrot, 1984, p. 43). Dans cette démocratie, ajoutera Tocqueville, la société peut faire tout ce qu'elle veut pour sa conservation et pour l'ordre établi. La plus grande liberté y règne et, comme s'il s'agissait là d'un corollaire, «le plus complet despotisme» dans les pénitenciers. La liberté n'est valable, en effet, que pour ceux qui acceptent l'ordre établi ou changeant, ou se trouvent dans des conditions qui leur permettent de le faire.

De fait, le pénitencier est contemporain du bouleversement rural et de l'économie agricole; il devance, en ce sens, l'industrialisation et la grande ville. Ce bouleversement n'en est pas pour autant la cause en soi (Ignatieff, 1981 , p. 164). C'est plutôt l'interprétation alarmiste du désordre (bouleversement) par les philanthropes réformistes qui agit, par le fond, sur les divers groupes de la société, dont ceux qui doivent créer les lois et les exécuter. La société civile est inquiète et elle accueille toute idée qui réduira ses inquiétudes. Ce «criminel», devenu ennemi du genre humain plus que simple vagabond, pauvre ou fou, qui incarne le mal, mérite désormais toute l'attention. Il exige, par ce qu'il représente, une punition capable de réconcilier intimidation et réforme, une punition qui calme les inquiétudes et qui rassure vis-à-vis de l'ordre à maintenir. Le pénitencier allie ainsi terreur et «humanité». Il n'attribue plus à la mauvaise administration toutes les tortures infligées jadis du fait de la négligence des anciens milieux carcéraux; il les incorpore plutôt dans ses règles afin de faire régner son autorité. Coupant le prisonnier du monde extérieur (et de tout autre monde), il tente de le refaire par une série d'artifices qui laissent leurs empreintes (mais pas celles qu'il recherche). 
Le pénitencier et la réforme qu’on y poursuit, à travers des débats acharnés sur le travail en isolement ou le travail en commun, semblent vouloir être plus qu'une copie de la discipline qu'exige l'usine comme telle. En effet, la discipline carcérale se veut davantage une technique de surveillance qu'une technique d'usage des corps au travail; c'est d'abord une discipline panoptique. Par la suite, cette discipline peut bien se faire omniprésente et s'exercer sur de plus grandes masses d'hommes, afin de réduire le travail non-productif (Gaudemar, 1982, pp. 18 et ss). Très difficile à instaurer à l'usine, cette discipline se bute aussi à de multiples échecs en prison, conduisant sans cesse à de nouvelles expériences, pour finalement aboutir à un amalgame complet.

L'Europe s'inspirera du modèle pennsylvanien (Cherry Hill), avant de proposer, un peu plus tard, son système progressif: régime d'emprisonnement cellulaire puis, ensuite, régime de travail en commun le jour. Cette formule combine ainsi les systèmes pennsylvanien et auburnien et assure une demiliberté au détenu soumis.

Ainsi, au Haut-Canada comme en Europe, l'emprisonnement tel qu'il se pratique aux États-Unis présente un modèle intéressant pour le traitement des "criminels». Cette mixture de travail et de réflexion doit pouvoir transformer et l'âme et le comportement d'une population d'endurcis, alors que les autres marginaux des classes dangereuses vivent des mesures plus douces.

Une première résolution, dans les débats de la Chambre du Haut-Canada, en vue de la construction du pénitencier de Kingston, apparaît en 1832. Le bâtiment n'est pas achevé, en 1835 , qu'on y accueille déjà les premiers détenus. Le pénitencier de Kingston assurera le cadre moral désiré, instituant la «pénitence» par l'entremise de l'isolement et de la correction, ainsi que du travail forcé. Le Haut-Canada va faire sien le traitement pénitentiaire, offrant la sécurité aux groupes qui se sentent en danger et réformant les mal-éduqués. Pour accéder à la «réforme morale», le Haut-Canada, comme les États-Unis, avait besoin de cette nouvelle institution: les anciennes prisons, servant de centres de détention pour ceux qui attendaient leur procès ou l'exécution de leur sentence, n'étaient pas adéquates pour effectuer la réforme des prisonniers (Beattie, 1977, p. 13). Pour réussir cette réforme, le prisonnier doit être livré pendant un certain temps au geôlier. Dès 1843, la législature du Canada-Uni adopte une loi en vertu de laquelle Kingston ne reçoit que des condamnés dont la peine est d'au moins deux ans. Jusque-là, le détenu pouvait y être pour une peine de six mois.

La construction du pénitencier a été modelée à partir du bloc cellulaire auburnien et du dôme pennsylvanien (Ciale, 1983, p. 19). L'installation d'un bloc cellulaire auburnien permettait la ségrégation des détenus le soir et l'association commune pour les travaux forcés le jour. L'installation d'un dôme 
pennsylvanien permettait une surveillance générale du pénitencier. La règle du silence était secondée par des punitions corporelles afin de renforcer la discipline générale du pénitencier. En somme, l'État, par la construction du pénitencier et la mise en place de ses principes, souhaitait créer un effet dissuasif général sur la population contre la commission de crimes futurs et visait la punition des coupables par l'incarcération et la réforme des détenus. Toutefois, les premières années d'opération du pénitencier de Kingston ne furent guère marquées de réussites.

Un directeur, Henri Smith, un directeur adjoint, William Powers (ancien directeur adjoint d'Auburn), cinq inspecteurs et un aumônier constituaient la direction générale du pénitencier, en 1835 . Dès leur première année en fonction, ils durent accueillir 55 prisonniers.

Mais c'est véritablement avec le rapport d'un comité spécial du gouvernement, en 1836, que le pénitencier connaîtra son coup d'envoi.

Selon le comité spécial pour l'adoption d'un système pénitentiaire efficace, la prévalence du crime dans le Bas-Canada nécessitait la construction d'un pénitencier. Mais, d'autre part, cette prévalence du crime était due principalement à l'absence de toute discipline dans les prisons, de même qu'à leur construction vicieuse, qui valait aux prisonniers d'être enfermés en commun.

Le comité spécial tentait ensuite d'expliquer que la société ne saurait avoir pour but de se venger d'un individu coupable de méfaits: «Son objet unique est d'empêcher le coupable de lui nuire dorénavant : elle croit parvenir à cette fin, soit en l'expulsant à jamais de son sein, soit en ne le séquestrant que pendant un temps limité, pendant lequel elle peut espérer son repentir et sa réforme. »

Le comité spécial rejette la réclusion pure et simple comme inefficace et comme ayant de funestes résultats, à savoir l'augmentation du crime à un degré alarmant. Pour le comité, la longueur de la réclusion, sa sévérité (supplices et tortures) n'ont eu pour résultat que la vengeance du coupable ou son insanité. Par contre, aux États-Unis, selon le comité spécial, on a mis fin à cette législation inepte en descendant «dans le cœur du coupable» et en troulvant «dans la cause même de son péché le moyen d'opérer sa réforme. L'oisiveté l'avait entraîné dans la débauche, de la débauche dans la misère, de la misère dans le crime, et du crime en prison. Il fallait donc pour le ramener à son devoir social lui donner les habitudes et le goût du travail.» (Bas-Canada, J.A.L., Appendice F.F.F., 1836, p. 2.)

Deux systèmes ont été élaborés aux États-Unis afin de donner le «goût du travail». Le comité spécial admire les deux, mais préfère celui d’Auburn à celui de la Pennsylvanie. Les raisons: les témoignages de Beaumont et de 
Tocqueville, selon lesquels il y aurait moins de mortalité, moins de frais. Le succès de Kingston et les témoignages de son directeur adjoint, W. Powers, les confirment dans leur opinion.

D'ailleurs, le président du comité spécial joint, à cet effet, une longue lettre de Powers adressée à L.-J. Papineau. Powers y vante la supériorité du système auburnien sur le système pennsylvanien.

Le grand avantage du système de Philadelphie, ainsi que ses partisans le prétendent, consiste en ce qu'il tend, plus que les autres, à produire la réforme dans le cour du Criminel. Si cela était vrai, et si son influence funeste sur la santé ne le proscrivait pas, il faudrait admettre que ce système est en effet le meilleur. Car le grand but d'un Pénitencier, après la sûreté publique, est assurément de réformer le coeur du Criminel. Mais je crois que cette théorie n'est pas vraie. Je pense que ce système est aussi peu propre à réformer les mours des Criminels, comparé au système d'Auburn, qu'il convient sous le rapport de la santé et des finances. Le trait saillant et caractéristique de ce système et par lequel on veut amener une réforme chez le Criminel est la réclusion et la solitude; c'est là le grand mobile. Il n'y a rien de tel que la solitude, disent les partisans de ce système, pour dompter l'esprit, adoucir les passions, et réformer le cœur.

Le 19 février 1836, Girod, un Suisse français, présente un témoignage qui renchérit sur celui de Powers:

Je sais que les partisans du système Pénitentiaire de Pennsylvanie prétendent changer entièrement leurs pupilles. Cette illusion est douce, mais néanmoins ce n'est qu'une illusion. Il faut un instant se mettre à la place du criminel, dans sa cellule solitaire, pendant quelques années peut-être, et se demander à quoi l'on ne serait pas disposé pour se soustraire le plus tôt possible à ce cruel état. Lisez l'histoire des Prisons d'État, de la Bastille, du Castel del Novo, etc., et vous aurez l'histoire future, et je crains l'histoire actuelle des pénitenciers de Philadelphie.

Je ne doute nullement que les Prisonniers ne s'endossent l'extérieur de la contrition, du repentir, de la religion; l'hypocrisie est si naturelle, et l'on peut dire si excusable dans leur situation!... Le système d'Auburn ne réussit pas non plus à changer le cœur des criminels; mais moins maltraités par la Société, plus protégés par elle dans leurs affections et leurs espérances, ils se soumettent à ses lois, parce que sans la soumission à ces lois ils savent très bien qu'ils ne sauraient rester dans cette Société qu'ils n'abhorrent pas, mais qu'ils regrettent, mais qu'ils rappellent de tous leurs vœux. (Bas-Canada, J.A.L., Appendice F.F.F, 1836.) 
Le comité spécial termine son rapport en souhaitant que la Chambre accorde son attention à un type d'institution auburnien tel qu'administré aux États-Unis.

La population carcérale de Kingston atteint le nombre de 150 détenus en 1838. De 1842 à 1845 , le nombre de prisonniers passe de 164 à 478 . Deux facteurs secondaires ont été suggérés pour expliquer l'augmentation soudaine de la population carcérale entre 1842 et 1845 ; l'admission, au pénitencier, des personnes condamnées du Bas-Canada, à partir de 1840 (acte d'Union), et celle des personnes condamnées sous le régime militaire (Beattie, 1977, p. 29). Malgré l'incidence de ces facteurs, la direction générale est amenée à rendre compte de la récidive des ex-détenus, vers la fin de 1847 . De plus, la direction générale fait l'objet de beaucoup de publicité néfaste concernant son administration et le gouvernement en est importuné. Aussi, en 1848, une commission (la commission Brown) est chargée de faire une enquête sur ce qui se passe au pénitencier de Kingston (Beattie 1977, p. 28).

La commission remet son rapport, en 1849 , au ministre de la Justice du gouvernement provincial du Haut-Canada. Le rapport prononce plusieurs chefs d'accusation contre la direction générale, spécialement à l'égard d'Henri Smith. Elle dénonce l'échec de la direction générale vis-à-vis de l'implantation de la réforme des criminels, de la discipline et de l'ordre. Selon cette commission, les principales causes de l'échec sont la conduite personnelle du directeur général (Henri Smith) et l'administration excessive des peines corporelles pour maintenir le régime du silence:

- rire et parler: 6 coups de chat-à-neuf-queues;

- se battre avec un détenu: 24 coups de chat-à-neuf-queues;

- parler à la toilette: 6 coups de nerf de bouf;

- fixer quelqu'un ou être distrait à table: régime au pain et à l'eau;

- laisser le travail: 36 heures en réclusion au pain et à l'eau dans une cellule sombre;

- parler français: 12 coups de nerf de bœuf (Gosselin, 1977, p. 52).

En 1845, 500 détenus reçoivent 2102 punitions; en 1847, on en administre 6063 . Un détenu reçoit en moyenne de 4 à 5 punitions corporelles en une année. Parfois, c'est par groupes de 20,30 ou 40 qu'hommes, femmes et enfants sont fouettés publiquement. On remarque qu'en 1846, par exemple, il y a 16 enfants en dessous de 16 ans et 75 adolescents entre 16 et 20 ans.

Enfants et adolescents sont fréquemment parmi les victimes du directeur et des gardiens. Un garçon de 10 ans, condamné pour 7 ans en 1845, fut fouetté 57 fois en huit mois et demi pour des offenses telles que rire et fixer quelqu'un. Un autre garçon de huit ans, Antoine Beauche, reçut 47 punitions corporelles 
en neuf mois. De même en fut-il pour des jeunes filles comme Sarah O'Connor, Elizabeth Breen. La liste des torturés n'en finit plus.

Si l'on en croit les commissaires,

(...) the penitenciary system during the Smith regime had been barbarous and cruel, as well as from the conviction that it had defeated rather than forwarded the ends of punishment. But the purpose of the pentienciary remained what it had been to create an environment in which men would be re-made (Beattie, 1977, pp. 34-35.)

Dans cette optique, la commission attribue l'échec du système pénitentiaire à des facteurs extérieurs à celui-ci, et permet alors un nouvel espoir visà-vis la réforme des criminels par l'entremise du pénitencier.

Pourtant, d'amples informations sont recueillies par les commissaires pouvant les entraîner à des conclusions fort différentes, qu'il s'agisse des questions de discipline, de surveillance, d'autoritarisme ou d'abus de pouvoir, toutes questions reliées expressément au "pénitencier et non à un homme».

Les commissaires rappellent que les objectifs du pénitencier tel qu'on le connaît en Angleterre sont de séparer les prisonniers de leurs anciens associés, de faire le tri entre ceux qui veulent se réformer et ceux qui ne le veulent pas, de leur enseigner des métiers et de leur faciliter ainsi la sortie. Les commissaires recommandent, à cette fin, que les détenus, à leur arrivée, soient soumis à un isolement complet, mais pour pas plus de six mois. Ensuite, ils pourront se joindre aux autres prisonniers au moment du travail et des repas, mais toujours en silence. Cette façon de faire serait la meilleure, car étant plus humaine, elle susciterait davantage la confiance du prisonnier et lui fournirait une bonne occasion d'effectuer sa réforme morale. Les commissaires tiennent aussi à ce que la direction du pénitencier laisse voir à ses pensionnaires qu'ils sont tous sur un pied d'égalité. Chacun des détenus devrait savoir à quoi s'attendre, connaître ses droits et obligations, lesquels doivent être définis très clairement. Enfin, pour assurer la «bonne marche» du pénitencier, des inspecteurs extérieurs devraient être nommés et investis d'un pouvoir réel.

Ce nouvel espoir de réforme ne se concrétise pas au niveau des conditions de vie à l'intérieur du pénitencier de Kingston mais il s'incarne, entre autres, dans la construction de nouveaux pénitenciers provinciaux: l'un à Saint-Jean, au Nouveau-Brunswick, et l'autre à Halifax, en Nouvelle-Écosse.

Ainsi, si nous retournons au pénitencier de Kingston quelque temps après les recommandations de réforme de la commission Brown, les inspecteurs, dont le statut et le rôle suggérés par cette même commission ont pris en principe une importance plus grande, décrivent la situation. 
Selon leurs dires, au moins la moitié des «crimes» pour lesquels le pénitencier était utilisé consistaient en des larcins, alors que l'autre crime le plus fréquent était le vol de cheval. Ils signalent ainsi les moyens excessifs utilisés par l'État dans le but de protéger de ces peccadilles une société qui n'est pas vraiment menacée par si peu.

L'inspecteur Nelson note, de plus, la conduite exemplaire des détenus alors que, par l'effet de la discipline très sévère, les châtiments sont fréquents. Les inconduites consistent en abus de langage, distractions, conversations, rires, etc., et sont punissables de plusieurs repas au pain et à l'eau, la privation de lit et le cachot. Le fouet est en usage dans les cas extrêmes et on n'outrepasse jamais 12 coups.

La réforme du coupable n'est pas réalisée au pénitencier, selon l'inspecteur. Encore en 1865, alors que Kingston avait en moyenne 700 détenus, 42 avaient été punis par le fouet, 6 mis à la chaîne, 399 au cachot, 39 en isolement, 45 privés de leur lit, 1395 réduits au pain et à l'eau (Boyer, 1966, p. 477).

\section{D'AUTRES PÉNITENCIERS ET DES PROBLÈMES SIMILAIRES}

L'acte de l'Amérique du Nord britannique renforce la division juridictionnelle en matière pénale, déjà énoncée dans la «règle de deux ans». Dès lors, il est clairement établi que le gouvernement fédéral est chargé de l'incarcération de tous les détenus purgeant des peines d'emprisonnement de deux ans et plus. Cette compétence permet au gouvernement fédéral de prendre en charge l'administration des pénitenciers de Kingston, Saint-Jean et Halifax. Par la suite, le gouvernement fédéral amorce un mouvement de construction de pénitenciers dans toutes les régions du pays, selon le modèle du pénitencier de Kingston (bloc cellulaire auburnien et dôme pennsylvanien): le pénitencier de Saint- Vincent-de-Paul, desservant la province de Québec, sauf les îles de la Madeleine, 1873; le pénitencier de Stoney-Mountain, desservant la province du Manitoba et les Territoires du Nord-Ouest du Canada, 1876; le pénitencier de la Colombie-Britannique, desservant la province de ce nom, 1878; le pénitencier de Dorchester, desservant les provinces de la Nouvelle-Écosse, du Nouveau-Brunswick et de l'île-du-Prince-Édouard ainsi que les îles de la Madeleine, 1880; le pénitencier d'Alberta, 1906 (fermé en 1920) et celui de la Saskatchewan, en 1911.

Lors de l'inauguration du pénitencier de Dorchester, le pénitencier de Saint-Jean et celui de Halifax tombent sous les compétences des autorités provinciales concernées.

Le pénitencier Saint-Vincent-de-Paul n'a vu le jour qu'en 1873, mais il en fut question de façon bien spécifique dès 1836 . Une conjoncture politique 
difficile, entre autres, en a retardé la construction (les troubles de 1837-1838 et leurs suites).

En somme, le pénitencier est, à ses débuts, un milieu d'une cruauté exceptionnelle: non pas une cruauté passive, comme dans les prisons où les détenus souffraient à cause des conditions de vie, mais bien une cruauté active où la torture est chose courante et où elle est institutionnalisée. Le scandale qu'il provoque n'est pas suffisant pour empêcher la diffusion de cette nouvelle pratique.

Il ne s'agira, avec tout cet équipement, que de perfectionner le système pour garder, catégoriser, réformer les "vrais criminels», catégorie dont sont écartés, en principe, les fous, les jeunes, les moins endurcis et les femmes en grande partie ${ }^{2}$. Mais, il devient évident que ce qu'un pénitencier ne pouvait faire, plusieurs pénitenciers ne le peuvent davantage. Bien plus, le désordre de l'un peut bien se transmettre à l'autre et prendre encore une nouvelle figure, multipliant ainsi le problème de la «réforme». Les pénitenciers deviennent de cette façon, comme les prisons d'ailleurs, des objets de réforme continue alors que, du même coup, ils n'apparaissent pas réformables. En effet, les enquêtes qui se suivent à partir de la fin du XIX ${ }^{e}$ siècle reprochent aux pénitenciers cela même que les premières enquêtes reprochaient.

Les vices des prisons ont pénétré tout entiers dans les pénitenciers et ce, pour y rester. Ces pénitenciers, cinquante ans après un premier essai, n'ont pu se corriger de leurs fautes originelles, dénoncées entre autres par Brown et les premiers inspecteurs.

Le pénitencier Saint-Vincent-de-Paul donne lieu à une enquête en 1898. Les commissaires y dévoilent le système de trafic entre les gardiens et les prisonniers, voire l'utilisation des prisonniers par les gardiens pour «voler» fournitures et nourriture à leurs propres fins... Des centaines de lettres cachetées, adressées aux prisonniers, demeuraient toujours, depuis des années, dans le bureau du geôlier. D'autres, ouvertes (par centaines encore) et brûlées par les prisonniers après qu'ils en eurent retiré l'argent, ne se rendirent jamais à leurs destinataires. Quasiment toutes les règles concernant la prévention de la malhonnêteté des employés ont été violées, conduisant à une quantité incroyable de pratiques fautives. La commission note enfin que 63 détenus (au 21 août) ont 21 ans et moins. Il faut, à cet effet, prendre des mesures, comme il a été dit si souvent. De même faut-il s'occuper du travail des prisonniers. Les commissaires terminent leur rapport en suggérant d'autres règles

2. Pour plus de détails sur cette question, voir dans Prison et ordre social au Québec la partie sur «Les femmes et les filles à préserver et à protéger». 
et en indiquant qu'il faudrait congédier un nombre considérable de personnes, du geôlier jusqu'aux gardiens.

La commission Macdonnell (1914) ne se contente pas de relever les irrégularités et infractions de toutes sortes dans les pénitenciers; elle insiste en quelque sorte sur la volonté de faire des réformes.

La journée d'un forçat l'incite, dès le départ, à vouloir modifier son traitement:

Une fois qu'on lui a désigné sa cellule, le forçat entre dans la vie routinière du pénitencier. Il se lève à six heures et demie du matin, fait sa toilette, fait son lit et à sept heures il sort pour son déjeuner. Ce déjeuner, il le reçoit dans une gamelle à mesure qu'il défile devant les guichets de service de la cuisine. De retour dans sa cellule, il mange son repas à l'aide d'une cuillère. L'usage de couteaux et de fourchettes est interdit. En passant pour se rendre au travail, il dépose son écuelle et sa cuillère à la cuisine. À midi, il prend son repas en se rendant à sa cellule; là, il déchire sa viande avec ses doigts, la mange, et rapporte l'écuelle à la cuisine lorsqu'il s'en retourne à son travail. Le soir, lorsqu'il quitte ses travaux, la même scène recommence. Il attrape son souper en se rendant à sa cellule où il reste jusqu'au lendemain matin. Le silence absolu doit régner pendant toute la journée. Il ne peut adresser la parole à un officier ou à un compagnon de bagne pendant les heures de travail «sauf par nécessité ou par rapport aux travaux». Il ne doit pas parler ni détourner la tête pendant qu'il est en rang et naturellement dans sa cellule, il n'a personne avec qui il puisse converser. C'est ainsi que la vie pénitentiaire d'un détenu se partage entre la cellule et le travail de l'atelier ou le tas de pierres, sans même une courte trêve à la monotonie des choses aux heures des repas. Il n'a pas le droit de garder des images ou des photographies de ses parents dans sa cellule, on ne lui permet pas d'avoir ou de lire un journal, mais on lui fournit des livres de la bibliothèque pénitentiaire. Lorsqu'il ne sait pas lire, il doit passer quatorze heures tous les jours, seul dans sa cellule, à dormir ou à réfléchir. Le dimanche, il passe toute la journée - à l'exception d'une heure pour le service religieux - dans sa cellule et lorsqu'une fête tombe un lundi, il l'observe par un supplément de réclusion cellulaire de vingt-quatre heures.

Un forçat dont la conduite est satisfaisante peut recevoir une fois tous les trois mois la visite de membres de sa famille et il peut leur écrire une fois tous les deux mois. Il peut recevoir des lettres de ses parents ou de ses amis, mais ces lettres doivent être brèves et consacrées exclusivement à des questions de famille ou d'affaires. On ne lui remet rien de ce que peuvent contenir ses lettres, coupures de journaux, insages, cartes, 
timbres, etc. Il n'est pas permis d'envoyer des cadeaux de Noël, des paniers ou des paquets de fruits, de la nourriture ou des pâtisseries au pénitencier. (Canada, Rapport de la Commission royale sur les pénitenciers; Document parlementaire, $\mathrm{n}^{\circ} 252,1914, \mathrm{pp} .8-9$.)

Comment un tel régime peut-il conduire à de meilleures dispositions? se demandent les commissaires.

Il faut repenser l'école, les règlements, les punitions. Il faut remplacer les châtiments corporels par la réforme morale quoique «si le châtiment du criminel est le seul but que poursuit la société, les pénitenciers du Canada remplissent toutes les conditions voulues» (Canada, Rapport de la Commission royale sur les pénitenciers, 1914 , p. 29; c'est nous qui soulignons).

Encore faut-il effectuer un bon classement car les détenus «comprennent toutes sortes de délinquants: le condamné pour première offense et le récidiviste, le criminel d'occasion et le criminel endurci; le garçonnet et le vieillard; l'athlète et l'invalide; le fin matois et l'imbécile» (Canada, Rapport de la Commission royale sur les pénitenciers, 1914, p. 32).

Enfin, selon les commissaires, le travail et la discipline pourraient aller de pair.

Biggar (président du comité nommé relativement à la révision des règlements pour les pénitenciers) remettra son rapport sept ans après celui de Macdonnell, soit en 1921.

La commission Biggar souligne qu'à part quelques amendements, la situation générale des pénitenciers est demeurée essentiellement la même depuis ses origines. Par exemple, la loi du silence est toujours de rigueur. Les prisonniers doivent travailler au moins dix heures par jour. Il n'y a pas de directives pour réglementer les modes de punition, que la commission juge cruelle. Le détenu n'a d'autre droit que celui d'être nourri et vêtu.

On note toutefois qu'aucune institution pénitentiaire n'applique vraiment les règlements. Des divergences très grandes se font remarquer d'une institution à l'autre dépendant des gardiens et de l'administration de chaque pénitencier. Par exemple, la loi du silence, qui est jugée incorrecte, n'est pas toujours appliquée. De plus, les détenus ne travaillent jamais plus de huit heures par jour en été et six, en hiver.

Pour Biggar et les membres de son comité:

À part la nourriture et les vêtements, le détenu est sans droits et la conduite prescrite est très automatisée; les sentiments sont interdits, ou du moins 
il lui est interdit de faire preuve d'émotions humaines. (Canada, Comité nommé..., 1921, p. 9; c'est nous qui traduisons.)

Le caractère vindicatif de la punition persiste et le système pénitentiaire possède toujours ses caractéristiques originales (...) (Canada, Comité nommé..., 1921, p. 11 ; c'est nous qui traduisons.)

La répression et la contrainte doivent faire place au développement et à la cure du prisonnier, selon ce comité qui s'applique à reformuler les règlements. Tout y passe, des devoirs du geôlier jusqu'à la classification, le travail, l'école et, bien sûr, la discipline. Une tendance commence à percer à travers les recommandations du comité, à savoir un traitement scientifique mais toujours fidèle aux maximes habituelles du carcéral.

\section{CONCLUSION}

Le pénitencier ne se distingue pas vraiment de la prison, sauf qu'on y reste un peu plus longtemps. La clientèle dans ses longs débuts est la même: des condamnés pour première offense, des infracteurs d'occasion, des femmes «en voie de se perdre», des jeunes garçons, des fous, des vieillards, des récidivistes. C'est le même individu (im)moral qui se retrouve dans les deux types de milieux carcéraux. Il n'est pas seulement semblable, il est identique en ce sens qu'il est plus ou moins apte au travail, qu'il est sans feu ni lieu et qu'il apparaît comme un fauteur de désordre.

Il est étonnant, à première vue, que l'on ait bâti deux types de structures pour appliquer le même traitement, sur le même individu, tout en affirmant que le pénitencier était le remède à la prison. Une recherche sans relâche de moralisation semble, entre autres, avoir permis cette doublure de la prison, alors même que cette moralisation semble s'imposer comme principe de gestion généralisé. Ainsi, pour que le pénitencier, en dépit de sa cruauté, fasse une telle percée à travers le Canada, comme dans tout l'Occident d'ailleurs, il ne doit sûrement pas constituer uniquement un moyen que l'État emprunte à l'Église. C'est par-dessus tout la plus-value de pouvoir que procure ce moyen, alors que la prison apparaît simplement comme une formule d'approche institutionnelle plus vaste. Elle sous-tend des fictions juridiques du même ordre, elle impose la même recherche de catégorisation, elle est à la poursuite d'un traitement qui lui échappe sans cesse. C'est ce dernier, d'ailleurs, qui réchappera non pas le criminel mais le pénitencier, semble-t-il. C'est son principe et les dispositifs qui s'ensuivent qui souvent se révèlent sauvegarder, non plus seulement le pénitencier (et la prison), mais la gouvernementalité qui s'installe, au tournant du siècle. 
En effet, la prison, déjà inscrite parmi nombre d'institutions de moralisation disciplinaire, dès les années 1830 et 1840 , obtient un succès extraordinaire compte tenu de ce qu'elle offre aux usagers, à leurs proches ainsi qu'au personnel. Elle apparait au moment où la logique capitaliste cherche à engendrer ses propres normes, dans lesquelles les classes s'insèrent. Appareils à éduquer, à travailler, à contrôler, à réprimer semblent tous répondre d'un droit positif. Ce droit dit neutre, dit scientifique s'enclenche plutôt dans un processus politique parce qu'il tend à la centralisation, intègre les différences dans l'unité du pouvoir étatique. Autrement dit, l'institution de la norme et les appareils pour la faire respecter sont posés en termes de lutte pour l'égalité alors qu'ils ont pour maître le promoteur même des différences entre les classes.

Cette insistance sur l'ordre en général et sur des ordres en particulier prend toute sa mesure dans la redéfinition des illégalismes, travail amorcé au début de XIX ${ }^{e}$ siècle et se poursuivant inexorablement, tout au cours du siècle. Cette redéfinition donne, entre autres, au "criminel» sa nature truquée alors que prend toute sa visibilité, à la fin du XIX ${ }^{e}$ siècle, l'arsenal de «pratiques juridiques qui construisent et rationalisent la gestion différentielle des illégalismes. C'est en tant que fiction que le droit et les institutions pénales constituent des instruments étatiques. Pluralismes des qualifications et pluralismes des formes procédurales peuvent ainsi être envisagés comme techniques de gouvernement, instruments de pouvoir tangibles dont l'analyse permet de rompre avec les approches essentialistes de l'État» (Lascoumes, 1986, p. 89).

C'est dans cet exercice de séparation des illégalismes que se fonde encore la domination d'une classe par rapport à une autre. Les illégalismes contre les biens forment une catégorie pénale «nette» et une répression est assurée par les tribunaux ordinaires et par les châtiments. Les illégalismes de droit forment une catégorie "pluriforme et non exclusivement pénale et une gestion plus ou moins différenciée assurée par des juridictions spéciales avec transactions, accommodements, amendes atténuées» (Lascoumes, 1986, p. 13). La domination peut s'imposer de par la catégorisation de l'illégalisme, sa qualification et la forme de règlement imposé. Elle peut mettre ainsi en évidence l'illégalisme des classes populaires et occulter, du même coup, celui des classes dominantes, entraînant une façon particulière de voir et de penser.

Cette pensée, associée à l'apparition du «discours fondamental de la criminologie» (Tulkens, 1986, p. 39) autour de la personnalité criminelle et de la dangerosité, permet l'entrée de la «défense sociale» (et la sortie du droit pénal classique), à la fin du XIX ${ }^{\mathrm{e}}$ siècle, en Europe occidentale. À ce moment, surgissent des menaces liées à l' "apogée du libéralisme et de l'industrialisation, à la crise économique, aux affrontements politiques et aux conflits sociaux... Politique familiale (mariage, logement), politique morale (alcoo- 
lisme, vagabondage), politique sociale (syndicats, corporations, mutuelles, assurances), politique pénale (avec comme cible notamment les ouvriers grévistes et les anarchistes) constituent ensemble des tentatives de réponses aux menaces...» (Tulkens, 1986, p. 39). Tout le corps social est touché et l'ennemi est présent partout, ce qui pèse lourd dans la demande répétée, sociale et politique, de protection et de répression. C'est tout ce processus qu'on pourrait encore appeler «défense sociale»: une espèce de projet de société pour s'assurer contre tout danger. Le Québec fera sien ce projet.

Ainsi, une notion de risque naît, notion qui permet de «concevoir un droit de l'État» sans pour autant y associer l'idée de faute et de responsabilité. Cette permission que se donne l'État lui accorde, du même coup, toute la flexibilité pour contrer le mal avant même qu'il n'arrive. Il lui permet de protéger et de défendre la société contre les dangers qui l'assaillent ou qui pourraient l'assaillir. Assurer la société contre les risques, voilà le nouveau projet social qui permet à l'État de se ressaisir à travers cette «gouvernementalité» moderne. En ce qui concerne le «criminel», risque parmi tant d'autres à l'intérieur du corps social à gouverner, on peut dorénavant le rendre pénalement responsable d'un acte sans juger de sa liberté à le commettre, mais en le liant au risque de criminalité qui s'insère dans sa personnalité (criminelle). C'est ce risque qu'il faudra faire disparaître en réchappant sa personnalité.

La responsabilité pénale, qui retrace la culpabilité dans la subjectivité de «criminel», ouvre la porte au traitement scientifique du criminel, fait place à la psychiatrie, à la criminologie, à la psychologie et au service social. Cette transformation du criminel en objet à risque, ce potentiel de traitement, cette opportunité pour la science qu'il garantit prennent forme dans les années 1940 au Québec. Les profils qu'on trace des individus dangereux, les mesures des risques en cause, de même que les explications et les pratiques qui en découlent apparaissent encore fréquemment comme l'exécution de ce projet de défense sociale. Ils soulèvent ainsi les mêmes interrogations que le projet lui-même.

\section{RÉFÉRENCES}

BEATTIE, J. M. (1977), Attitudes Towards Crime and Punishment in Upper Canada, 1830-1850: A Documentary Study, Toronto, University of Toronto, Centre of Criminology.

BOYER, R. (1966), Les Crimes et les châtiments au Canada français de XVII au XX' siècle, Ottawa, Le Cercle du livre de France.

CANADA (1898), Rapport de la Commission royale d'enquête sur le pénitencier SaintVincent-de-Paul, Ottawa, Documents de la session, $\mathrm{n}^{\circ} 18$. 
CANADA (1914), Rapport de la Commission royale d' enquête sur les pénitenciers, Ottawa, Imprimeur du Roi, Document parlementaire, $n^{\circ} 252$.

CANADA (1921), Ministère de la Justice, Comité nommé relativement à la révision des règlements pour les pénitenciers et des modifications à apporter à la loi régissant les pénitenciers, Ottawa, Imprimeur du Roi.

CIALE, J. (1983), The Development of Canadd's Prisons and Penitentiaries, Ottawa, Université d'Ottawa, Département de criminologie.

FECTEAU, J.-M. (1980), Vers une étude de la crise des appareils de répression au BasCanada: La Prison de Québec, 1814-1834, Québec (inédit).

FOUCAULT, M. (1975), Surveiller et punir. Naissance de la prison, Paris, Gallimard.

FOUCAULT, M. (1980), «La poussière et le nuage», dans M. Perrot (éd.), L'Impossible Prison, Paris, Seuil, pp. 29-39.

GAUDEMAR, J.-P. (1982), L'Ordre et la production, Paris, Dunod.

GOSSELIN, L. (1977), Les Pénitenciers: un système à abattre, Montréal, Éditions coopératives Albert Saint-Martin.

IGNATIEFF, M. (1981), «State, Civil Society and Total Institution: A Critique of Recent Social Histories of Punishment», dans Crime and Justice: An Annual Review of Research, vol. III, pp. 153-192.

LAPLANTE, J. (1989), Prison et ordre social au Québec, Ottawa, Presses de l'Université d'Ottawa.

LASCOUMES, P. (1986), «Le grondement de la bataille» Actes, vol. 54, pp. 84-89.

LEFEBVRE, F (1954), «La vie à la prison de Montréal aux XIX ${ }^{\mathrm{e}}$ siècle», dans Revue d' histoire de l'Amérique française, vol. VII, $\mathrm{n}^{\circ} 4$, pp. 524-537.

PERROT, M. (1984), «Introduction aux ceuvres de Tocqueville», dans A. Tocqueville, Écrits sur le système pénitentiaire en France et à l'étranger, Paris, Gallimard.

TULKENS, F (1986), «Droit pénal, criminologie et sciences pénitentiaires à la fin du $\mathrm{XIX}^{e}$ siècle" Actes, vol. 64, pp. 38-41. 